\section{Discrete-Time Supervisory Control of Families of Two-Degrees-of-Freedom Linear Set-Point Controllers}

Donato Borrelli, A. Stephen Morse, and Edoardo Mosca

\begin{abstract}
This paper describes a discrete-time "high-level" controller, called a "supervisor," capable of switching into feedback with a discretetime single-input/single-outpu (SISO) system, a sequence of linear twodegrees-of-freedom (2-DOF) set-point controllers. Each controller is selected among a family of candidates so as to cause the output of the system to approach and track a constant reference input. It is shown that the proposed supervisor can stabilize the loop and ensure a zero set-point tracking error even if constant load disturbances are present.
\end{abstract}

Index Terms - Amdaptive control, discrete-time systems, supervisory control, switching, uncertain linear systems.

\section{INTRODUCTION}

In this paper we describe and analyze the discrete-time version of a simply structured "high-level" controller called a "supervisor," which is capable of switching into feedback, with a discrete-time linear time-invariant single-input/single-output (SISO) system, a sequence of two-degrees-of-freedom (2-DOF) linear controllers from a family of candidate controllers so as to cause the output of the system to approach and track a constant reference input. The supervisor orchestrates the selection of each controller according to a certainty equivalence-based decision strategy, in that the controller chosen to be put in feedback is the one which has the best idea of what the system is and therefore should be able to do the best job of controlling the system.

Earlier studies about similar problems are in [1]-[4], while an extensive work on the continuous-time version of supervisory control studied in this paper is in [5]. In fact, [5] can be considered our starting point, the aim of this paper being the development of a discrete-time formalization of a supervisory control problem similar to the one of [5]. However, here our interest is in dealing with 2-DOF controllers, whereas in [5] only 1-DOF controllers are considered. The discrete-time setting allows one to get further insight into the behavior of digitally implementable supervisors. The relevance of 2-DOF control has been known for a long time to practitioners. Here, thanks to properties of discrete-time algorithms and to appropriate reconfiguration of the 2-DOF control system, it is possible to prove asymptotic stability and tracking for the supervisory controller in the presence of constant load disturbance.

The paper is organized as follows. After the problem formulation in Section II, in Section III the main features of the supervisory control system are described. Closed-loop system behavior is analyzed in Section IV for a system subject to constant load disturbances, and it is shown that the proposed supervisor guarantees globally bounded states as well as zero-offset tracking. Some concluding remarks are made in Section V.

Manuscript received May 20, 1996.

D. Borrelli and E. Mosca are with the Dipartimento di Sistemi e Informatica, Università di Firenze, 50139 Firenze, Italy.

A. S. Morse is with the Department of Electrical Engineering, Yale University, New Haven, CT 06520 USA.

Publisher Item Identifier S 0018-9286(98)09442-2.

\section{Problem Formulation}

Throughout this paper, we assume that the system to be controlled is a discrete-time SISO linear time-invariant system $\Sigma_{P}$ with control input $u$ and controlled output $y$. We further assume that $\Sigma_{P}$ 's transfer function from $u$ to $y$ is a member of a known class of admissible transfer functions $\mathcal{C}_{P}$ of the form $\mathcal{C}_{P}=\left\{\nu_{p}(d): p \in \mathcal{P}\right\}$, where $\mathcal{P}$ is $\mathcal{C}_{P}$ 's parameter space which may be either a finite set of indexes, or a closed bounded subset of a real finite-dimensional linear space, and $\nu_{p}(d) \triangleq\left(\beta_{p}(d) / \alpha_{p}(d)\right)$ is a preselected strictly causal nominal transfer function. It is assumed that for each $p \in \mathcal{P}, \beta_{p}(d)$ and $\alpha_{p}(d)$ are coprime polynomials in the unit backward-shift operator $d$. Moreover, prompted by the requirements of set-point control, it is assumed that $\nu_{p}(d)$ are transfer functions nonzero at $d=1$. The specific model of the system to be controlled is given by the following equation:

$$
\alpha_{p^{*}}(d) y(t)=\beta_{p^{*}}(d) u(t)+\boldsymbol{d}(t)
$$

where $d$ is a bounded disturbance, and $p^{*}$ is a fixed but unknown element of $\mathcal{P}$. Let us consider the problem of making $y$ track any constant reference input $r(t) \equiv r$. Toward this end we introduce a tracking error

$$
\boldsymbol{e}_{\boldsymbol{T}}(t) \triangleq r-y(t)
$$

and an integrating subsystem to generate $u$; i.e.,

$$
u(t)=u(t-1)+\delta u(t) .
$$

Here $\delta u$ is the one-step control increment.

We take now as given, an indexed family $\mathcal{K} \triangleq\left\{\kappa_{q}(d): q \in \mathcal{P}\right\}$ of 2-DOF [6] causal controller transfer functions of the form $\kappa_{q}(d)=$ $\left[\left(\gamma_{q}(d) / \rho_{q}(d)\right)\left(\xi_{q}(d) / \rho_{q}(d)\right)\right]$ where for each $q \in \mathcal{P},\left[\gamma_{q}(d) \xi_{q}(d)\right]$ and $\rho_{q}(d)$ are pairs of coprime polynomials. Each of these pairs of transfer functions is used for generating the control increment $\delta u$

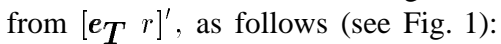

$$
\rho_{q}(d) \delta u(t)=\gamma_{q}(d) e \boldsymbol{T}^{(t)}+\xi_{q}(d) r .
$$

Furthermore, the following properties are assumed to hold.

Zero-Offset Property: $\xi_{q}(1)=0, q \in \mathcal{P}$.

Stability Margin Property: There is a positive number $\lambda_{S} \in[0,1)$ s.t. for each $p \in \mathcal{P}$, the closed-loop poles (see Fig. 1)

$$
(1-d) \rho_{p}(d) \alpha_{p}(d)+\gamma_{p}(d) \beta_{p}(d) \in \mathbb{R}_{\lambda_{S}}[d]
$$

with $\mathbb{R}_{\lambda_{S}}[d]$ the set of polynomials whose zeros lie in $\{z \in C:|z| \geq$ $\left.\lambda_{S}^{-1}\right\}$.

Assumption 1: $\mathcal{C}_{P}$ and $\mathcal{K}$ have the property that $\kappa_{p}=\kappa_{q}$ for each pair $p, q \in \mathcal{P}$ at which $\nu_{p}=\nu_{q}$.

Assumption 2: There exist integers $n_{\nu}$ and $n_{\kappa}$ so that for every $p, q \in \mathcal{P}$, the McMillan degrees of $\nu_{p}$ and $\kappa_{q}$ are at most $n_{\nu}$ and $n_{\kappa}$, respectively.

The problem of interest is to develop a supervisory logic for switching into the feedback loop between $\left[\boldsymbol{e}_{\boldsymbol{T}} r\right]^{\prime}$ and $\delta u$ a sequence of 2 -DOF controllers with transfer functions in the set $\mathcal{K}$, so as to achieve satisfactory closed-loop "performance." By satisfactory performance we mean the following. If $r$ and $\boldsymbol{d}$ are, respectively, a constant and bounded reference and load disturbance, then for all possible initializations, it follows that: 1) Global Boundedness: $\delta u, u$, and $y$ are bounded responses and 2) Tracking and Disturbance Rejection: $e_{T} \rightarrow 0$. In developing a supervisory control algorithm which achieves the above objectives, other practical requirements 


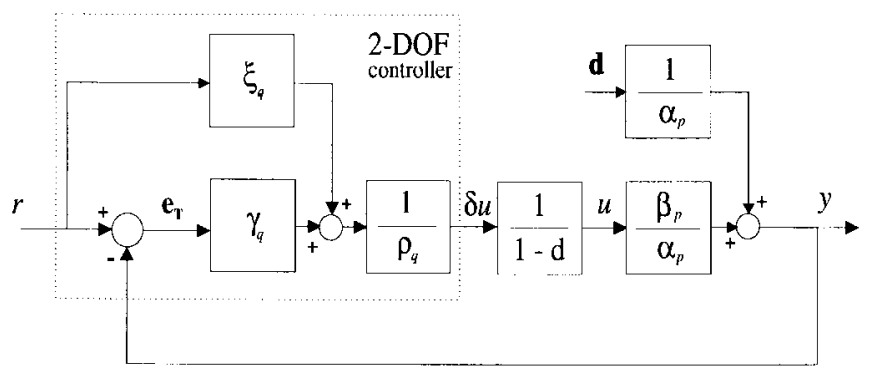

Fig. 1. 2-DOF feedback-feedforward interconnection.

are taken into consideration: the supervisory controller is required to ensure satisfactory performance without the aid of a probing signal; the selection of the controllers does not follow any prerouted search through the admissible controller set. ${ }^{1}$

\section{State-Space Systems AND Supervisor}

State-Space Systems: In the following it is shown how to construct a convenient realization of one component of the overall control system, consisting of state-shared estimators and of a family of parameterized linear state-shared controllers. This realization is particularly useful in the sequel for stability analysis. To this end, what we want to do is to specify a doubly indexed system $\Sigma_{p q}$, $p, q \in \mathcal{P}$ whose inputs are $e_{T}, y, \delta u$, and $r$ and outputs are $e_{p}$ and the $q$ th "candidate control signal" $\delta u_{q} . \Sigma_{p q}$ is to have the following properties.

1) If the transfer function of the system $\Sigma_{P}$ were $\nu_{i}$, and $i=p$, and if $\boldsymbol{d}$ were a zero signal, then no matter what the definition of $\delta u, e_{p}$ would tend to zero as fast as $\lambda_{S}^{t}$.

2) The transfer function from $\left[\boldsymbol{e}_{\boldsymbol{T}} r\right]^{\prime}$ to $\delta u$ under the feedback connection $\delta u_{q}=\delta u$ should be $\left[\left(\gamma_{q} / \rho_{q}\right)\left(\xi_{q} / \rho_{q}\right)\right]$, after cancellation of possible common factors in $\mathbb{R}_{\lambda_{S}}[d]$.

The construction of $\Sigma_{p q}$ is as follows. Pick monic polynomials $\omega_{\nu}$ and $\omega_{\kappa}$ in $\mathbb{R}_{\lambda_{S}}[d]$ of degrees less than or equal to $n_{\nu}+1$ and $n_{\kappa}$ respectively. Then, first pick SISO reachable pairs $\left(\bar{A}_{\nu}, \bar{b}_{\nu}\right),\left(\bar{A}_{\nu \kappa}, \bar{b}_{\nu \kappa}\right),\left(\bar{A}_{\kappa}, \bar{b}_{\kappa}\right)$ in such a way that $\omega_{\nu}$, the least common multiple between $\omega_{\nu}$ and $\omega_{\kappa}$, and $\omega_{\kappa}$ are the characteristic polynomials of $\bar{A}_{\nu}, \bar{A}_{\nu \kappa}$, and $\bar{A}_{\kappa}$, respectively. The three pairs above are of orders $n_{\nu}+1, n_{\nu}+n_{\kappa}+1$, and $n_{\kappa}$, respectively.

Second, define $A_{C}=$ block diagonal $\left\{\bar{A}_{\nu}, \bar{A}_{\nu \kappa}, \bar{A}_{\kappa}, \bar{A}_{\kappa}\right\}, d_{C}=$ $\left[\begin{array}{llll}\bar{b}_{\nu}^{\prime} & 0 & 0 & 0\end{array}\right]^{\prime}, b_{C}=\left[\begin{array}{llll}0 & \bar{b}_{\nu \kappa}^{\prime} & 0 & 0\end{array}\right]^{\prime}, h_{C}=\left[\begin{array}{lllll}0 & 0 & \bar{b}_{\kappa}^{\prime} & 0\end{array}\right]^{\prime}, i_{C}=\left[\begin{array}{llll}0 & 0 & 0 & \bar{b}_{\kappa}^{\prime}\end{array}\right]^{\prime}$. Third, define $c_{p}, f_{q}, g_{q}$, and $h_{q}$ to be the unique solutions ${ }^{2}$ to the equation

$$
\begin{gathered}
{\left[\begin{array}{l}
c_{p} \\
f_{q}
\end{array}\right]\left(I-d A_{C}\right)^{-1} d\left[d_{C} b_{C} h_{C} i_{C}\right]+\left[\begin{array}{ccc}
0_{2 \times 2} & 0 & 0 \\
& q_{q} & h_{q}
\end{array}\right]} \\
{\left[\begin{array}{cccc}
\frac{\omega_{\nu}-(1-d) \alpha_{p}}{\omega_{\nu}} & \frac{\beta_{p}}{\omega_{\nu}} & 0 & 0 \\
0 & \frac{\omega_{\kappa}-\rho_{q}}{\omega_{\kappa}} & \frac{\gamma_{q}}{\omega_{\kappa}} & \frac{\xi_{q}}{\omega_{\kappa}}
\end{array}\right] .}
\end{gathered}
$$

Finally, define $\Sigma_{p q}$ to be the following parameter-dependent system:

$$
\begin{aligned}
x_{C}(t+1)= & A_{C} x_{C}(t)+d_{C} y(t)+b_{C} \delta u(t) \\
& +h_{C} e_{T}(t)+i_{C} r \\
\delta u_{q}(t)= & f_{q} x_{C}(t)+g_{q} e_{T}(t)+h_{q} r \\
e_{p}(t)= & c_{p} x_{C}(t)-y(t) .
\end{aligned}
$$

\footnotetext{
${ }^{1}$ By a prerouted search we mean an algorithm which sequentially steps through the admissible controller set along a predetermined path or route.

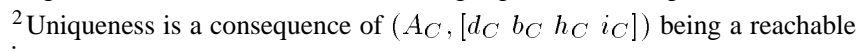
pair.
}

We assume to be in closed loop whenever the feedback connection $\delta u_{q}=\delta u$ is effective. It is quite clear from the definition of $\Sigma_{p q}$ that in this case $\left[\left(\gamma_{q} \omega_{\kappa} / \rho_{q} \omega_{\kappa}\right)\left(\xi_{q} \omega_{\kappa} / \rho_{q} \omega_{\kappa}\right)\right.$ will be the reducible transfer function of $\Sigma_{p q}$ from $\left[\boldsymbol{e}_{\boldsymbol{T}} r\right]^{\prime}$ to $\delta u$. In view of the definitions of $\omega_{\kappa}$, the property 2) above is satisfied. It is proven in [7] that property 1) is satisfied as well. Moreover, it can be easily checked that the parameter-dependent system (6)-(8) satisfies the requirement that $\kappa_{p}=\kappa_{q}$ whenever $p$ and $q$ are points in $\mathcal{P}$ for which $\nu_{p}=\nu_{q}$. Prompted by property 2) of $\Sigma_{p q}$, we now define $\Sigma_{C}$ to be the dynamical system (6)-(8) where $q=\sigma$ is a piecewise-constant "switching signal" taking values in $\mathcal{P}$, and $\sigma(t)$ is the output of the "supervisor."

Supervisor: The supervisor is a specially structured hybrid dynamical system whose output is a switching signal $\sigma$ taking values in $\mathcal{P}$. The supervisor specifies at each time which controller in the set $\mathcal{K}$ has to be connected in the feedback loop between $\left[\begin{array}{ll}\boldsymbol{e}_{\boldsymbol{T}} & r]^{\prime}\end{array}\right.$ and $\delta u$. The supervisor considered here consists of a performance weight generator $\Sigma_{W}$ and a dwell time switching logic $\Sigma_{D} \cdot \Sigma_{W}$ is a dynamical system which, on the basis of the output prediction of each state-shared estimator $c_{p}$ and the real time data $x_{C}$ and $y$, via (8) produces a performance signal $\pi_{p}$ associated with each nominal model $\nu_{p} p \in \mathcal{P}$, as follows:

$$
\pi_{p}(t+1)=\lambda^{2} \pi_{p}(t)+e_{p}^{2}(t), \quad p \in \mathcal{P}, \lambda \in[0,1] .
$$

The dwell time switching logic $\Sigma_{D}$ [5] is an event-driven system that continuously performs the minimization of the $\pi_{p}$ on $\mathcal{P}$ and accordingly selects the best index $\sigma \in \mathcal{P}$ for the controller to switch in the loop. The dwell time is the minimum number of time samples that must elapse between two successive switching times. This integer $\tau_{D}$ must satisfy the condition $\tau_{D} \geq \tau_{C}$, where $\tau_{C}$ represents the computation time for the minimization procedure of the $\pi_{p}$. The dwell time logic operates as follows. Consider a time $t=\bar{t}$ at which $\tau$ is reset to zero and increased up to $\tau_{D}$ according to $\tau(t+1)=\tau(t)+1$. Suppose also that at $\bar{t}, \sigma(\bar{t})$ has switched to a value $\sigma_{0}$. Then

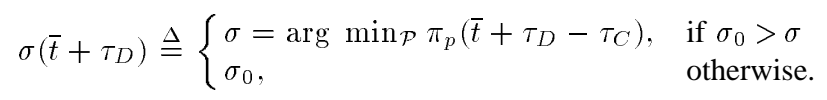

If $\sigma_{0}>\sigma, \tau$ is reset to zero and the process is repeated to compute the next value $\sigma\left(\bar{t}+2 \tau_{D}\right)$; otherwise, if $\sigma_{0} \leq \sigma, \sigma$ does not switch at $t=\bar{t}+\tau_{D}, \tau$ is reset to $\tau_{D}-\tau_{C}$, a new minimizer $\sigma\left(\bar{t}+\tau_{D}+\tau_{C}\right)=\arg \min \mathcal{P} \pi_{p}\left(\bar{t}+\tau_{D}\right)$ is computed, and so on. For a more detailed discussion of the supervisor the reader is referred to [5] or [8].

\section{ExACT MATCHING}

Since extensions to the case of an infinite $\mathcal{P}$ can be derived with little effort, in the following we restrict the analysis to the case of $\mathcal{P}$ being a finite set. Our aim now is to analyze the closed-loop behavior of the supervisory control system described above for the case when nominal transfer function $\nu_{p^{*}}(d)$ matches or equals that of system model $\Sigma_{P}$ for a fixed but unknown value of $p^{*} \in \mathcal{P}$. Moreover, in (1) we assume that $\boldsymbol{d}$ is a constant. These assumptions enable us to develop certain basic results which shall be used in sequels to this paper to analyze the control system under more realistic assumptions. In order to exploit the same analysis tools described in previous papers (see [5] for the continuous-time 1-DOF case and [7] and [8] for the discrete-time 1-DOF case), our aim at this point shall be to rewrite (6)-(8) describing the system $\Sigma_{C}$ in alternative form. Fix the set-point value $r$ and solve

$$
\overline{x_{C}}=A_{C} \overline{x_{C}}+d_{C} y+b_{C} \delta u+h_{C} \boldsymbol{e}_{\boldsymbol{T}}(t)+i_{C} r
$$


where $y=r, e_{T}=0$, and $\delta u=0$. This gives $\overline{x_{C}}=(I-$ $\left.A_{C}\right)^{-1}\left[d_{C}+i_{C}\right] r$. In this way, we are selecting an equilibrium state $\overline{x_{C}}$ which is bounded and corresponding to which set-point tracking with zero-offset error is achieved. For each triple of points $p, q, l$ in $\mathcal{P}$, define now matrices

$$
\begin{aligned}
d_{q} & =g_{q} b_{C}+h_{C}-d_{C} ; \quad f_{q l}=f_{q}-g_{q} c_{l} \\
c_{p l} & =c_{p}-c_{l} ; \quad A_{q l}=A_{C}+\left(d_{C}-h_{C}\right) c_{l}+b_{C} f_{q l}
\end{aligned}
$$

and accordingly a shifted state $x(t)=x_{C}(t)-\overline{x_{C}}$. Using (2) and (6)-(8) together with (10) and (11), it is straightforward to verify that for all $p, l \in \mathcal{P}$ and switching signals $\sigma \in \mathcal{P}$

$$
\begin{aligned}
x(t+1) & =A_{\sigma l} x(t)+d_{\sigma} e_{l}(t) \\
\delta u_{\sigma}(t) & =f_{\sigma l} x(t)+g_{\sigma} e_{l}(t) \\
e_{p}(t) & =c_{p l} x(t)+e_{l}(t) \\
e_{T}(t) & =e_{l}(t)-c_{l} x(t)
\end{aligned}
$$

initialized from some $x(0)=x_{C}(0)-\overline{x_{C}}$. In deriving these equations in addition to $f_{q}\left(I-A_{C}\right)^{-1}+h_{q}=0, q \in \mathcal{P}$ (zero-offset property), the following identities have been used: $c_{p}\left(I-A_{C}\right)^{-1} d_{C}=1, c_{p}(I-$ $\left.A_{C}\right)^{-1} i_{C}=0, p \in \mathcal{P} ; f_{q}\left(I-A_{C}\right)^{-1} d_{C}=0, q \in \mathcal{P}$. These are direct consequences of the derivation of (6)-(8) for the parameterdependent system $\Sigma_{p q}$. Hereafter follows a concise description of the stability analysis of the overall supervisory control system considered in this paper (for all the details see [7]).

\section{A. Convergence}

Fix the initial values of the integrator state $u$, controller state $x_{C}$, and supervisor's state variables and $\sigma$. By virtue of the exact matching assumption and the fact that $\mathcal{P}$ is a finite set, there can be shown to exist a nonempty finite set $\mathcal{P}^{*}$, with $\mathcal{P}^{*} \triangleq\left\{p: \Sigma_{t=0}^{\infty} e_{p}^{2}(t)<C^{*}, C^{*} \geq\right.$ $0, p \in \mathcal{P}\}$, such that beyond a certain finite time $t^{*}, \sigma$ takes values only in $\mathcal{P}^{*}$ [7]. This allows one to rewrite (12) in the following output-injection form holding for every $t \geq t^{*}$ (take $\left.l=p^{*}\right)$ :

$$
x(t+1)=\left(A_{\sigma p^{*}}+K_{\sigma} C\right) x(t)-K_{\sigma} \bar{e}(t)+d_{\sigma} e_{p^{*}}(t)
$$

where $e_{p^{*}}$ and $\bar{e} \triangleq C x$ are signals with finite $\ell^{2}\left(\mathcal{Z}_{+}\right)$norms ${ }^{3}$; ( $\left.C, A_{\sigma p^{*}}\right)$, thanks to the stability margin property (4) (see [5], [7] for details), are detectable pairs for each $\sigma \in \mathcal{P}^{*} ; K_{\sigma}$ is any appropriately sized, bounded matrix; $\sigma(t)$ is the switching signal.

Our aim now is to prove that, for some suitably defined function $K_{\sigma}$, the linear time-varying system (16) is exponentially stable, ${ }^{4}$ that is, the state transition matrix $\Phi$ of $A_{\sigma p^{*}}+K_{\sigma} C$ satisfies $|\Phi(t, \mu)| \leq \lambda_{0}^{(t-\mu-a)}$ for $t \geq \mu \geq 0, \lambda_{0} \in[0,1)$ and $a \geq 0$. Here and from now on all the matrices norms $|\cdot|$ are assumed to be submultiplicative. The following result provides a way of showing that an inequality of this type holds also in this case.

Theorem 1 (Switching Theorem): Let $\lambda_{0} \in[0,1)$ and $\tau_{0} \geq n$ be fixed. Let $\left(C_{q_{0} \times n}, A_{n \times n}, B_{n \times m}\right)$ be a left invertible system. Suppose that $\left\{\left(C, F_{p}\right): p \in \mathcal{P}\right\}$ is a closed bounded subset of matrix pairs in $\mathbb{R}^{q \times n} \oplus \mathbb{R}^{m \times n}$ with the property that for each $p \in \mathcal{P}$. $\left(C, A+B F_{p}\right)$ is detectable with stability $\operatorname{margin}^{5} \lambda_{0}$. There exist a constant $a \geq 0$ and a bounded matrix-valued output injection function $p \mapsto K_{p}$ on $\mathcal{P}$ which, for any switching signal $\sigma: \mathcal{Z}_{+} \rightarrow \mathcal{P}$ with dwell time not smaller than $\tau_{0}$, causes the state transition matrix of $A+B F_{\sigma}+K_{\sigma} C$ to satisfy

$$
|\Phi(t, \mu)| \leq \lambda_{0}^{(t-\mu-a)}, \quad t \geq \mu \geq 0 .
$$

${ }^{3}$ In this note a signal $f(t)$ has a finite $\ell^{2}\left(\mathcal{Z}_{+}\right)$norm iff $\Sigma_{t=0}^{\infty} f^{2}(t)<\infty$. ${ }^{4}$ Note that $A_{\sigma p^{*}}+K_{\sigma} C$ is a time-varying matrix, since $\sigma(t)$ is the output of the supervisor.

${ }^{5} \mathrm{~A}$ matrix pair $(C, A)$ is detectable with stability margin $\lambda \in[0,1)$, if $\left(C, \mu^{-1} A\right)$ is a detectable pair for every $\lambda<\mu<1$.
The proof of this theorem can be found in [7] but is not given here as it closely follows the similar one given in [5] for the continuoustime case. Before explaining how the switching theorem applies to the problem under consideration, another result, called the Squashing lemma, will be illustrated. In contrast with the theorem, the proof of the latter lemma exhibits some significant differences with respect to the continuous-time case. The Squashing lemma is proved in the Appendix.

Squashing Lemma: Let $\left(C_{q_{0} \times m}, A_{n \times n}\right)$ be a fixed constant observable matrix pair, and let $\tau_{0}$ be a positive number s.t. $\tau_{0} \geq n$. For each positive number $\delta$ there exists a positive number $\lambda \in[0,1)$, and a constant output-injection matrix $K$ for which

$$
\left|(A+K C)^{t}\right| \leq \delta \lambda^{\left(t-\tau_{0}\right)}, \quad t \in \mathcal{Z}_{+} .
$$

The lemma states that in the discrete-time case in order to fulfill inequality (18), which in turn is used to derive (17), it is sufficient to choose any $\tau_{D}$ greater than or equal to $n$, rather than just greater than zero, as suffices for continuous-time. In other words, in discrete-time it is not possible to switch arbitrarily fast, but only with dwell time not smaller than $n$, with $n$ being the order of a state-space realization of the closed-loop supervisory control system.

Unfortunately, for the applicability of the Squashing lemma, the observability of the matrix pair $\left(C, A_{\sigma p^{*}}\right)$ is required, while for the supervisory control problem at hand, only detectability is ensured. Nevertheless, in the Switching theorem's proof it is possible to exploit the particular algebraic structure of the problem so as to be able of applying the Squashing lemma. The application of the Switching theorem is as follows. Take $A_{p p^{*}}=A+B F_{p}$, since according to (11), $A_{p p^{*}}=\left[A_{C}+\left(d_{C}-h_{C}\right) c_{p}^{*}\right]+b_{C} f_{p p^{*}}$. Then identify $\mathcal{P}$ with $\mathcal{P}^{*}$, each $C_{p}, p \in \mathcal{P}$ with $C, \tau_{0}$ with $\tau_{D}$, and pick $\lambda_{0} \in\left[\lambda_{S}, 1\right)$. The switching theorem's two main hypotheses, regarding detectability of the matrix pairs $\left(C, A_{p p^{*}}\right)$ and left invertibility of $\left(C, A_{p p^{*}}, B\right)$, are fulfilled. In fact, the first is a direct consequence of the stability margin property, while the second depends on the particular definition of $\mathcal{P}^{*}$. The theorem states that there exists a bounded output injection matrix, namely $K_{p}$, which exponentially stabilizes $A_{\sigma p^{*}}+K_{\sigma} C$. Consider now (16). Since the Switching theorem ensures the validity of condition (17), it follows that $x(t)$ would have a finite $\ell^{2}\left(\mathcal{Z}_{+}\right)$ norm. Therefore $x_{C}(t)$ and $\delta u(t)$ [by virtue of (13)], tend to finite limits (more precisely to $\overline{x_{C}}$ and to zero respectively), and as a consequence $y(t)$ tends to $r$ and $u(t)$ tends to a finite limit. The latter would be true because of the converging of $\boldsymbol{d}, y(t)$ and $\delta u(t)$ to constant values and because $\Sigma_{P}$ 's transfer function is nonzero at $d=1$. Now let $\Sigma$ denote the closed-loop supervisory control system consisting of $\Sigma_{P}$, tracking error $\boldsymbol{e}_{\boldsymbol{T}}(t)$ defined by (2), integrating subsystem (3), system $\Sigma_{C}$ defined by (6)-(8), with $\delta u(t)=\delta u_{\sigma}(t)$, dwell time switching supervisor as after (9), where the weighting parameter ${ }^{6} \lambda$ is chosen in such a way that $\lambda \in\left[\lambda_{S}, 1\right)$. The following result holds.

Theorem 2: Let $\tau_{D}$ be any number such that $\tau_{D} \geq n \triangleq \operatorname{dim}\left(A_{C}\right)$, and $A_{C}$ as before (5). Suppose that $\Sigma_{P}$ 's transfer function equals the nominal system model transfer function $\nu_{p}(d)$ for some $p=p^{*} \in \mathcal{P}$. Then for each constant set-point value $r$, each constant disturbance $\boldsymbol{d}$, and each initial state $\left\{u(0), x_{C}(0), \sigma(0)\right\}, y(t) \rightarrow r$, and $x_{C}(t)$ and $u(t)$ tend to finite limits as $t \rightarrow \infty$.

\section{CONCluding Remarks}

The discrete-time version of the "high level" supervisory controller introduced in [5] has been devised and analyzed. Switching is performed by a logic which selects controllers among a family of

${ }^{6} \lambda$ plays an important role in the supervisor; any reader interested in the meaning of $\lambda$ should refer to [7] or [8]. 
fixed gain candidate controllers by means of comparing in real time normed output estimation errors. In contrast with [5] and [8] which deal with 1-DOF controllers, however, here we have considered the situation of switching amongst 2-DOF controllers. As is well known, 2-DOF controllers have potentially superior tracking performance. It has been shown that in the absence of unmodeled system dynamics, the proposed discrete-time supervisor can successfully perform its function, provided that the dwell time $\tau_{D}$ is larger than a quantity related to an upper bound of the McMillan degree of the system. This is true even if constant load disturbances are present. Issues of future investigation are the analysis in the presence of bounded and possibly time-varying load disturbances as well as unmodeled dynamics.

\section{APPENDIX}

Proof of the Squashing Lemma: Given the matrix pair $(C, A)$ observable, $\left(A^{\prime}, C^{\prime}\right)$ is reachable. Then, without loss of generality we can assume that such a pair is in an $n$-dimensional canonical reachability form, i.e.,

$$
A^{\prime}=\left[\begin{array}{cccc}
0 & & & \\
\vdots & & I_{n-1} & \\
0 & & & \\
-a_{n} & \cdots & & -a_{1}
\end{array}\right]
$$

According to the standard synthesis procedure, let us suppose that we want to get via state-feedback, the closed-loop characteristic polynomial $\hat{\chi}(d), \hat{\chi}(d)=\Pi_{i=1}^{n}\left(1-d \lambda \lambda_{i}\right)$, where $\lambda$ is a number in $[0,1)$ and $\lambda_{i} \neq \lambda_{j} \in[0,1), i \neq j=1, \cdots, n$. Thus, we can determine a feedback vector $F=\left[\begin{array}{lll}f_{n}(\lambda) & \cdots & f_{1}(\lambda)\end{array}\right]$ with $f_{i}(\lambda)$ real polynomials of degree less than or equal to $n$. We can write the corresponding feedback injection matrix

$$
\hat{A}^{\prime}=A^{\prime}+C^{\prime} F=\left[\begin{array}{cccc}
0 & & & \\
\vdots & & I_{n-1} & \\
0 & & & \\
f_{n}(\lambda)-a_{n} & \cdots & \cdots & f_{1}(\lambda)-a_{1}
\end{array}\right]
$$

which, in turn, is similar to the following diagonal matrix:

$$
T^{-1} \hat{A}^{\prime} T=\Lambda=\operatorname{diag}\left\{\lambda \lambda_{i}, i=1, \cdots, n\right\} .
$$

Taking this into account and considering (20), it is easy to see that $T$ is the Vandermonde matrix

$$
T=\left[\begin{array}{cccc}
1 & \cdots & \cdots & 1 \\
\lambda \lambda_{1} & \cdots & \cdots & \lambda \lambda_{n} \\
\left(\lambda \lambda_{1}\right)^{2} & \cdots & \cdots & \left(\lambda \lambda_{n}\right)^{2} \\
\vdots & \vdots & \vdots & \vdots \\
\left(\lambda \lambda_{1}\right)^{n-2} & \cdots & \cdots & \left(\lambda \lambda_{n}\right)^{n-2} \\
\left(\lambda \lambda_{1}\right)^{n-1} & \cdots & \cdots & \left(\lambda \lambda_{n}\right)^{n-1}
\end{array}\right] .
$$

Looking at the structure of $T$ it is not difficult to find that

$$
\operatorname{det} T=j\left(\lambda_{1}, \cdots, \lambda_{n}\right) \lambda^{n(n-1) / 2}
$$

where $j\left(\lambda_{1}, \cdots, \lambda_{n}\right)$ is a linear combination of powers of $\lambda_{1}, \lambda_{2}, \cdots, \lambda_{n}$. Further, $T^{-1}=\operatorname{Adj}(T)(\operatorname{det} T)^{-1}$. From (21) it follows that

$$
\left|\hat{A}^{\prime t}\right|=\left|T \Lambda^{t} T^{-1}\right| \leq\left|T\left\|\Lambda^{t}\right\| T^{-1}\right|
$$

Our aim is to make $\lambda$ small enough in order to satisfy

$$
\left|T^{-1}\right|\left|\Lambda^{t}\right||T| \leq \delta \lambda^{\left(t-\tau_{0}\right)}
$$

Therefore, since $|T|$ tends to a constant as $\lambda$ goes to zero, we must use $\left|\Lambda^{t}\right|$ to kill the negative power of $\lambda$ appearing in $\left|T^{-1}\right|$. It is not difficult to realize that the highest negative degree of $T^{-1}$ will appear in the last column. This highest negative degree is given by

$$
\begin{aligned}
& -\frac{(n-1)(n-2)}{2}+\operatorname{deg}[\operatorname{det} T] \\
& \quad=\frac{(1-n)(n-2)}{2}+\frac{n(n-1)}{2}=n-1 .
\end{aligned}
$$

Hence $\left|T^{-1}\right|$ will be dominated by a term of the type $\lambda^{-(n-1)}$. Consequently

$$
\left|T^{-1} \| \Lambda^{t}\right||T| \leq\left|T^{-1}\right|\left(\lambda_{\max } \lambda\right)^{t}|T|
$$

where $\lambda_{\max } \triangleq \max _{i}\left\{\lambda_{i}\right\}$. Now as $\lambda \rightarrow 0$, the upper bound in (27) approaches $c \cdot \lambda^{t-n+1} \lambda_{\max }^{t}$, where $c$ is a positive constant. The latter can be made smaller than $\delta \lambda^{\left(t-\tau_{0}\right)}$, with $\delta$ an arbitrary number s.t. $\delta \in(0,1)$, by choosing a sufficiently small nonzero $\lambda$, provided that $\tau_{0} \geq n$. So (24) and (25) together with the fact that $\left|\left(A^{\prime}+C^{\prime} F\right)^{t}\right|=\left|\left(A+F^{\prime} C\right)^{t}\right|$, and with the choice $K=F^{\prime}$, imply that

$$
\left|(A+K C)^{t}\right|<\delta \lambda^{\left(t-\tau_{0}\right)}, \quad t \geq 0 .
$$

Therefore (18) is true.

Remark: The proof of the Squashing lemma can be simplified by taking the gain matrix $K$ above so that $A^{\prime}+C^{\prime} K^{\prime}$ is nilpotent (supervisor subsystems with dead-beat dynamics) and choosing $\lambda$ as follows:

$$
\begin{aligned}
\lambda<\min \left\{1, \delta^{1 / \tau_{0}},\left(\frac{\delta}{\left|A^{\prime}+C^{\prime} K^{\prime}\right|}\right)^{1 /\left(\tau_{0}-1\right)}, \cdots\right. \\
\left.\cdot\left(\frac{\delta}{\left|\left(A^{\prime}+C^{\prime} K^{\prime}\right)^{n-1}\right|}\right)^{1 /\left(\tau_{0}-n+1\right)}\right\} .
\end{aligned}
$$

This implies that (18) holds.

\section{REFERENCES}

[1] B. Mårtensson, "The order of any stabilizing regulator is sufficient a priori information for adaptive stabilization," Syst. Contr. Lett., vol. 6 , no. 2, pp. 87-91, 1985.

[2] M. Fu and B. R. Barmish, "Adaptive stabilization of linear systems via switching controls," IEEE Trans. Automat. Contr., pp. 1079-1103, Dec. 1986.

[3] S. J. Cusumano and K. Poolla, "Adaptive control of uncertain systems: A new approach," in Proc. American Automatic Control Conf., June 1988, pp. $355-359$.

[4] D. E. Miller, "Adaptive control of uncertain systems," Ph.D. dissertation, Univ. Toronto, 1989.

[5] A. S. Morse, "Supervisory control of families of linear set-point controllers-Part 1: Exact matching," IEEE Trans. Automat. Contr., vol. 41, pp. 1413-1431, Oct. 1996.

[6] E. Mosca, Optimal, Predictive, and Adaptive Control. Englewood Cliffs, NJ: Prentice-Hall, 1995.

[7] D. Borrelli and A. S. Morse, "Discrete-time supervisory control of families of linear set-point controllers," DSI, Univ. di Firenze, Tech. Rep. no. 22/95, 1995.

[8] _ _ "Discrete-time supervisory control of families of linear set-point controllers," in Proc. 13th IFAC World Congr., San Francisco, CA, July 1996, vol. K, pp. 211-216. 